\title{
Calculating Dynamic Time Quantum for Round Robin Process Scheduling Algorithm
}

\author{
Aashna Bisht \\ Department of Computer \\ Science, Jamia Hamdard \\ New Delhi, India
}

\author{
Mohd Abdul Ahad \\ Department of Computer \\ Science, Jamia Hamdard \\ New Delhi, India
}

\author{
Sielvie Sharma \\ Department of Computer \\ Science, Jamia Hamdard \\ New Delhi, India
}

\begin{abstract}
Process scheduling using Round Robin Algorithm makes use of a fixed amount of time quantum that is assigned to each process to complete its execution. If the process is able to complete its execution within this time period, it is discarded from the ready queue otherwise it goes back to the rear of the ready queue and waits for its next turn. In this paper we have presented amelioration to the conventional round robin algorithm by evaluating the time quantum on the basis of the burst times of the various processes waiting in the ready queue. The allocation of dynamic time quantum to various processes will have a direct impact on various performance parameters like turnaround time, waiting time, response time and the number of context switches. Further we are increasing the time quantum for the processes which require a fractional more time to complete their execution than the allocated time quantum cycle(s).
\end{abstract}

\section{Keywords}

Context Switches, Scheduling, Turnaround time, Wait time.

\section{INTRODUCTION}

Process scheduling may be defined as the policies and mechanism to allocate CPU to various processes [11], [12]. The fundamental aim of the operating system is to discharge the task of process management and its performance can be construed in terms of how well it administers different jobs coming to the CPU for execution [11], [12], [14]. A good scheduling policy ensures that the most crucial job(s) gets the intended resource (CPU time)[11], [12], [13]. The effectiveness of a processor can be inspected using the following parameters [11], [12], [13], [14].

\subsection{Turnaround Time}

It is defined as the time taken by the processes since their commencement till they execute completely [11], [17].

\subsection{Wait Time}

It is defined as the time consumed by the processes waiting in ready queue till the CPU is allocated to them [11], [12].

\subsection{Throughput}

It is defined as the rate of execution of the total number of processes [11], [12], [17].

\subsection{Context switches}

It is defined as the swapping of CPU between the processes [11], [12], [14], 1[7].

\subsection{Response time}

It is the total time since the acceptance for a request of the
CPU until the first reaction [11], [12].

Few common Scheduling Algorithms include First Come First Serve (FCFS), Shortest Job First (SJF) and Round Robin (RR) algorithms [11], [12], [13], [14], [15], [17]. FCFS algorithm gives the CPU to the job that arrives first at the CPU for execution. The job is not preempted until it completes its execution [11], [12], [13], [17]. Shortest Job First algorithm gives the CPU to the process with the minimal burst time first, here also the job is not preempted until it completes its execution [11],[12], [13], [17]. Round Robin algorithm is philosophically similar to the FCFS algorithm however in this algorithm every job is assigned a fixed time quantum to complete its execution [11] [12], [13], [15], [17].

\section{PRELIMINARIES}

Round robin scheduling algorithm is one of the most researched algorithms of process scheduling. The Researchers of [1] have proposed a new NK algorithm which calculates the time quantum using the average of the burst times of the processes. The authors of [2] introduced a pipelining concept which improves the efficiency of CPU. Their philosophy was to divide a process into sub processes and each will execute in conjunction with other sub processes. The researchers of [3] have talked about the extension to time quantum only for those processes whose remaining time is equal to or less than the assigned threshold value. Here threshold value is taken as quarter of time quantum. The Novelists of [4] have figured out improvement in the average waiting time with the help of proposed SJRR CPU scheduling algorithm. The authors of [5] highlighted the problem with conventional round robin algorithm that jobs are preempted even if small amount of execution time is left for a job, and they have proposed a modification based on the harmonic mean concept. The novelists of [6] have suggested to divide the processes in ready queue into two catagories .Time quantum will be modified for the process in first category and the process in second category will be processed and executed same as conventional round robin algorithm. The authors of [7] proposed a modification in conventional round robin algorithm for real time and embedded systems. They have calculated an intelligent time slice in their approach. The researchers of [8] proposed a modified round robin approach using the concept of fuzzy logic. The novelists of [9] proposed a median based approach to find the modified value of time quantum. The authors of [17] calculated the mean of the burst times of the processes and then find the difference between the individual burst time and the calculated mean. Finally they allocated the process with the largest difference to the CPU. The Novelists of [18] talked about fuzzy rule based round robin scheduling algorithm wherein they made use of three priority queues and user defined priorities. They also made use of the ratio of the remaining time to the burst 
time of the processes. The authors of [19] compared various proposals on improving the conventional round robin algorithm.

\section{PROPOSED APPROACH}

In our approach we are finding the optimum value of the time quantum on the basis of the burst times of the jobs waiting in the ready queue. The primary difference of our approach from the conventional round robin algorithm is that the time quantum is prefixed in the conventional algorithm but in our approach we are dynamically calculating the value of the time quantum. The figure given below represents the philosophy of our proposed approach. The various abbreviations used in our Proposed Approach are: TQ: Time Quantum, NQC: Number of Quantum cycle, BT: Burst Time, RT: Remaining Time

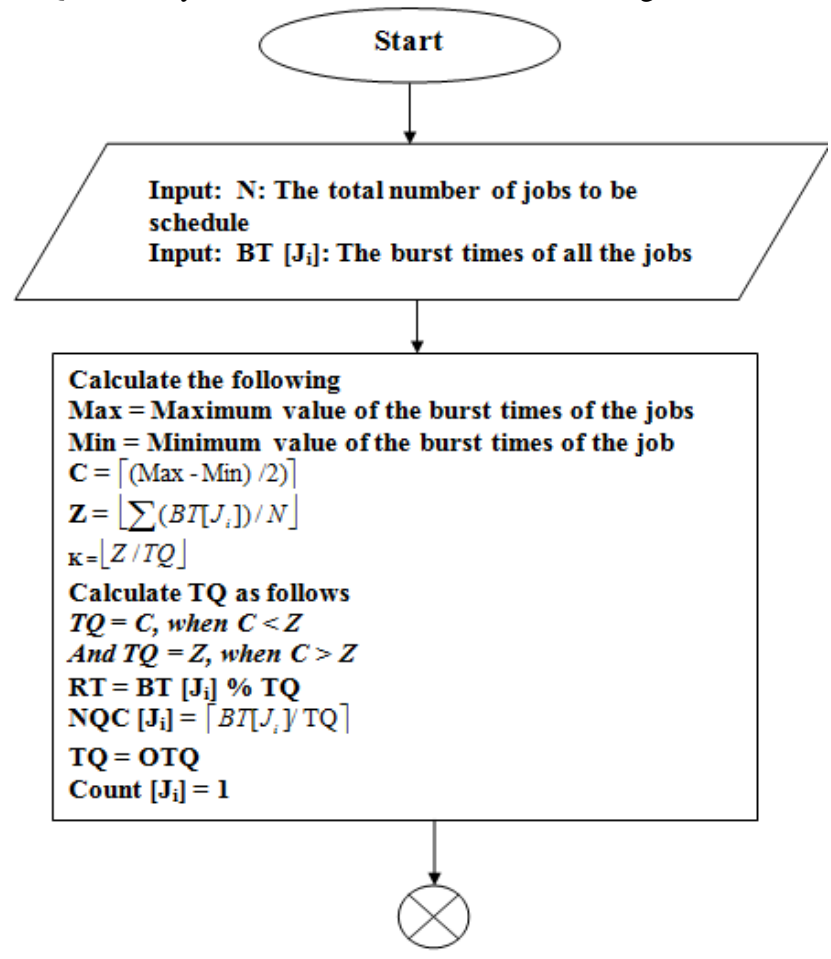

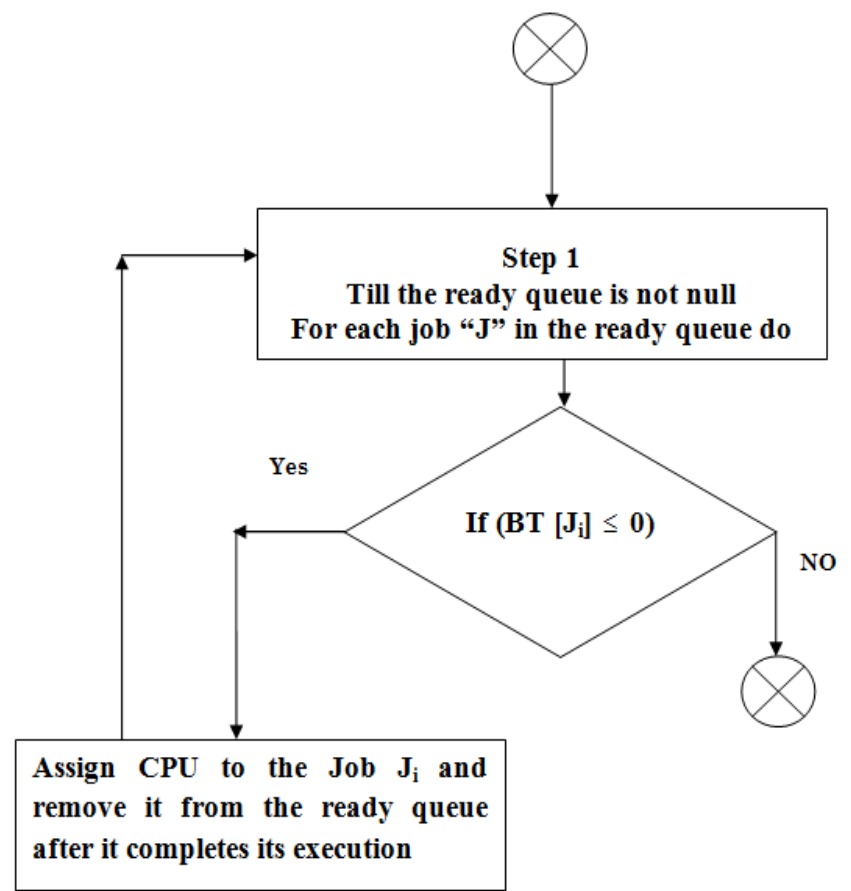

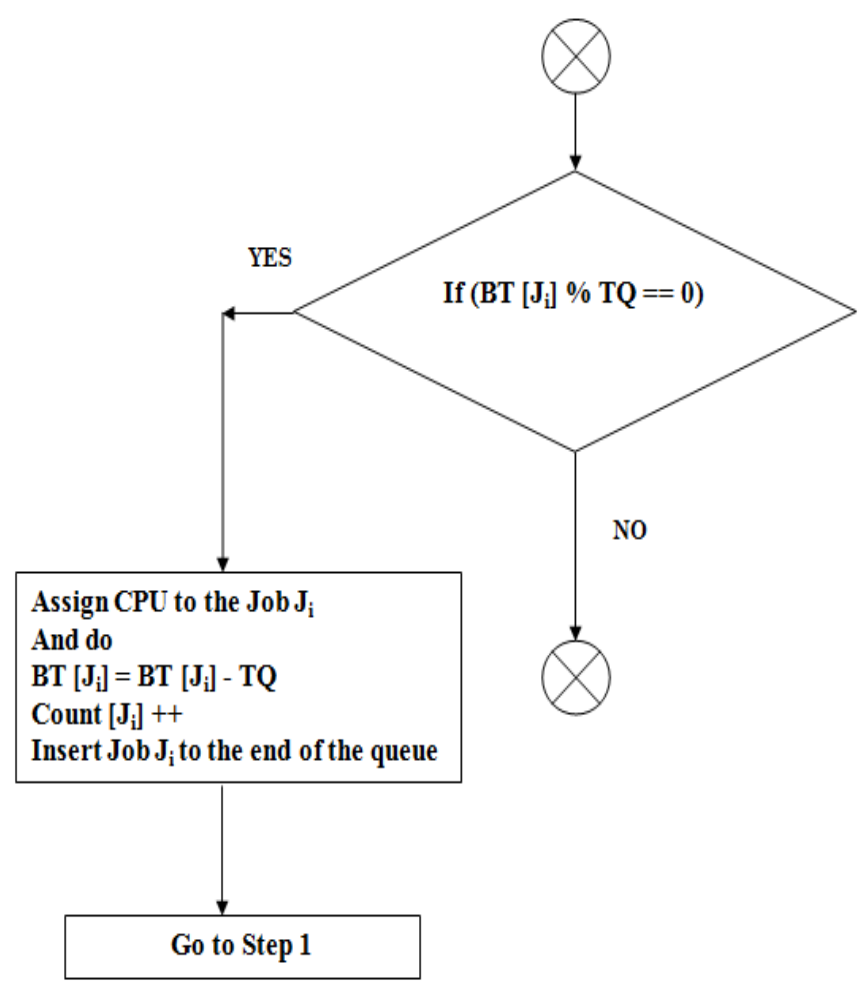




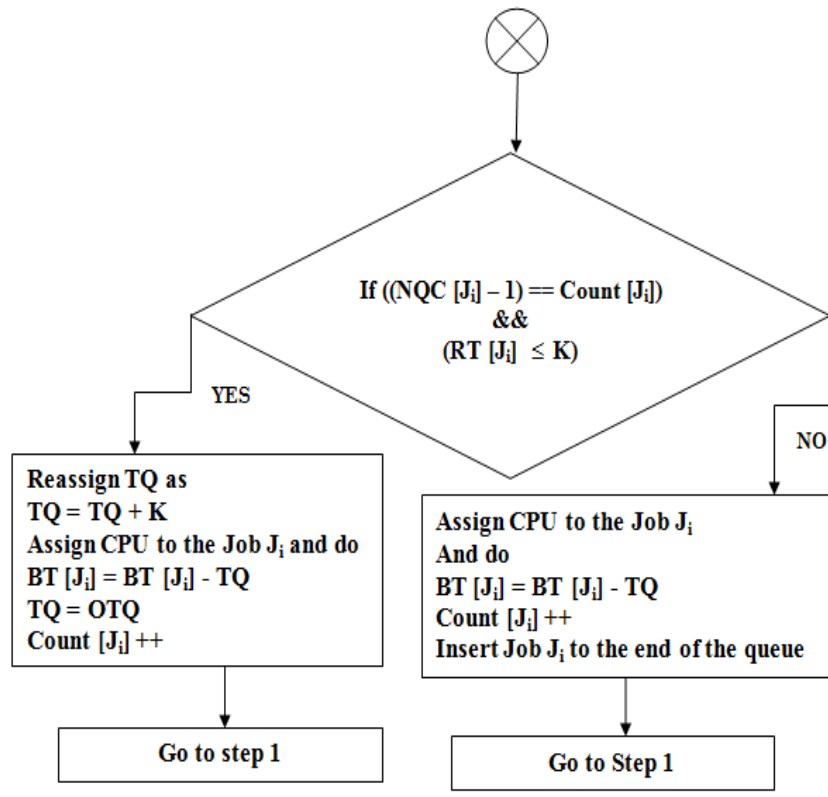

Fig 1: Proposed Approach

\section{HYPOTHETICAL EXAMPLES}

This section provides few hypothetical examples to show the working of the proposed approach as well as its comparison with the conventional round robin approach

\subsection{Example 1}

Table 1: Jobs Table with burst times for Conventional Round Robin Approach

\begin{tabular}{|c|c|}
\hline \multicolumn{2}{|c|}{ Time Quantum (TQ) $=\mathbf{5}$} \\
\hline Job Name $\left(\mathbf{P}_{\mathbf{i}}\right)$ & Burst Time BT[Pi] \\
\hline P1 & 19 \\
\hline P2 & 09 \\
\hline P3 & 23 \\
\hline P4 & 15 \\
\hline P5 & 16 \\
\hline
\end{tabular}

\begin{tabular}{|c|c|c|c|c|c|c|c|c|c|}
\hline P1 & P2 & P3 & P4 & P5 & P1 & P2 & P3 & P4 & P5 \\
\hline
\end{tabular}

\begin{tabular}{|l|l|l|l|l|l|l|l|l|}
\hline P1 & P3 & P4 & P5 & P1 & P3 & P5 & P3 \\
\hline 49 & 54 & 59 & 64 & 69 & 73 & 78 & 79 & 82
\end{tabular}

Fig 2: Gantt chart using Conventional Round Robin Algorithm

\subsubsection{Calculations using the Classical Round Robin Algorithm:}

Average Turnaround Time

$=(73+34+82+64+79) / 5$

$=66.4$

Average Wait Time

$=((73-19)+(34-09)+(82-23)+(64-15)+(79-16)) / 5$

$=(54+25+59+49+63) / 5$

$=50$

Number of Context switches $=17$.

\subsubsection{Calculation using the proposed Approach}

Calculation of TQ:

$\operatorname{Max}(\mathrm{BT})=23$

$\operatorname{Min}(\mathrm{BT})=09$

$\mathrm{C}=\lceil([\operatorname{Max}(\mathrm{BT})-\operatorname{Min}(\mathrm{BT})] / 2)\rceil$

$=\lceil(23-09) / 2\rceil$

$=\lceil 14 / 2\rceil$

$=7$

$\left.\mathrm{Z}=\left\lfloor\sum(\mathrm{BT}[\mathrm{Pi}]) / \mathrm{N}\right)\right\rfloor=16$

As $\mathrm{C}<\mathrm{Z}$,

Therefore $\mathrm{TQ}=7$

Calculation of $\mathrm{K}$

$\mathrm{K}=\lfloor Z / T Q\rfloor$

$=\lfloor 16 / 7\rfloor$

$=2$

Table 2: Jobs Table with burst times for Proposed Approach

\begin{tabular}{|c|c|c|c|}
\hline \multicolumn{4}{|c|}{ Time Quantum (TQ) $=7$} \\
\hline $\begin{array}{c}\text { Job } \\
\text { Name } \\
\mathbf{P}_{\mathbf{i}}\end{array}$ & $\begin{array}{c}\text { Burst } \\
\text { Time } \\
\text { BT[Pi] }\end{array}$ & $\begin{array}{c}\text { Remaining Time } \\
\text { RT[Pi] } \\
\text { BT[Pi]\% TQ }\end{array}$ & $\begin{array}{c}\text { Number of } \\
\text { Cycles } \\
\text { NQC }\left[\mathbf{P}_{\mathbf{i}}\right]= \\
{\left[\mathrm{BT}\left[\mathrm{P}_{\mathrm{i}}\right] / \mathrm{TQ}\right]}\end{array}$ \\
\hline P1 & 19 & 5 & 3 \\
\hline P2 & 09 & 2 & 2 \\
\hline P3 & 23 & 2 & 4 \\
\hline P4 & 15 & 1 & 3 \\
\hline P5 & 16 & 2 & 3 \\
\hline
\end{tabular}

Now as per the proposed approach we will modify the time quantum for those processes for which the remaining time is less than or equal to $\mathrm{K}$. Other processes will be executed as per the conventional round robin algorithm with the original time quantum value. Here, those processes are P2, P3, P4, and P5 only. However, their time quantum will be increased only in the last but one CPU cycle. The figure given below depicts the Gantt chart using the proposed approach.

\begin{tabular}{|c|c|c|c|c|c|c|c|c|c|c|}
\hline P1 & P2 & \begin{tabular}{|l|} 
P3 \\
\end{tabular} & P4 & P5 & P1 & P3 & P4 & P5 & P1 & P3 \\
\hline
\end{tabular}

Fig 3: Gantt chart using Proposed Approach

Average Turnaround Time

$=(73+16+82+59+68) / 5$

$=59.6$

Average Wait Time

$=((73-19)+(16-09)+(82-23)+(59-15)+(68-16)) / 5$

$=(54+7+59+44+52) / 5$

$=43.2$

Number of Context switches $=10$.

\subsection{Example 2}

Table 3: Jobs Table with burst times for Conventional Round Robin Approach 


\begin{tabular}{|c|c|}
\hline \multicolumn{2}{|c|}{ Time Quantum(TQ) = 12} \\
\hline Job Name $\left(P_{i}\right)$ & Burst Time BT[Pi] \\
\hline P1 & 21 \\
\hline $\mathrm{P} 2$ & 16 \\
\hline P3 & 29 \\
\hline P4 & 44 \\
\hline P5 & 30 \\
\hline
\end{tabular}

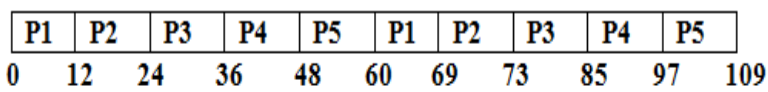

\begin{tabular}{|l|l|l|l|}
\hline P3 & P4 & P5 & P4 \\
\hline
\end{tabular}

$\begin{array}{lllll}109 & 114 & 126 & 132 & 140\end{array}$

Fig 4: Gantt chart using Conventional Round Robin Algorithm

\subsubsection{Calculations as per Classical Round}

Robin Algorithm:

Average Turnaround Time

$=(69+73+114+140+132) / 5$

$=528 / 5$

$=105.6$

Average Wait Time

$=((69-21)+(73-16)+(114-29)+(140-44)+(132-30)) / 5$

$=388 / 5$

$=77.6$

Number of Context switches $=13$.

\begin{tabular}{|c|c|}
\hline \multicolumn{2}{|c|}{ Time Quantum $=\mathbf{4}$} \\
\hline Process Name $\left(\mathbf{P}_{\mathrm{i}}\right)$ & Burst Time $\left(\mathbf{B T}\left[\mathbf{P}_{\mathrm{i}} \mathbf{]}\right)\right.$ \\
\hline P1 & 19 \\
\hline P2 & 14 \\
\hline P3 & 21 \\
\hline P4 & 26 \\
\hline P5 & 14 \\
\hline
\end{tabular}

\subsubsection{Calculation as per proposed Approach}

$$
\begin{aligned}
& \operatorname{Max}(\mathrm{BT})=44 \\
& \operatorname{Min}(\mathrm{BT})=16 \\
& \begin{aligned}
\mathrm{C} & =\lceil([\mathrm{Max}(\mathrm{BT})-\operatorname{Min}(\mathrm{BT})] / 2)\rceil \\
& =\lceil(44-16) / 2\rceil \\
& =\lceil 28 / 2\rceil \\
& =14 \\
\mathrm{Z} & =\left\lfloor\left(\sum(\mathrm{BT}[\mathrm{Pi}]) / \mathrm{N}\right)\right\rfloor=28
\end{aligned} \\
& \text { As } \mathrm{C}<\mathrm{Z},
\end{aligned}
$$

\begin{tabular}{|c|c|c|c|}
\hline \multicolumn{5}{|c|}{ Time Quantum (TQ) $=\mathbf{1 4}$} \\
\hline $\begin{array}{c}\text { Job } \\
\text { Name } \\
\mathbf{P}_{\mathbf{i}}\end{array}$ & $\begin{array}{c}\text { Burst } \\
\text { Time } \\
\text { BT[Pi] }\end{array}$ & $\begin{array}{c}\text { Remaining Time } \\
\text { RT[Pi]= } \\
\text { BT[Pi]\% TQ }\end{array}$ & $\begin{array}{c}\text { Number of } \\
\text { Cycles } \\
\text { NQC }\left[\mathbf{P}_{\mathbf{i}}\right]= \\
\left\lceil\mathrm{BT}\left[\mathrm{P}_{\mathrm{i}}\right] / \mathrm{TQ}\right]\end{array}$ \\
\hline P1 & 21 & 7 & 2 \\
\hline P2 & 16 & 2 & 2 \\
\hline P3 & 29 & 1 & 3 \\
\hline P4 & 44 & 2 & 4 \\
\hline P5 & 30 & 2 & 3 \\
\hline
\end{tabular}

Therefore $\mathrm{TQ}=14$

Calculation of $\mathrm{K}$

$\mathrm{K}=\lfloor Z / T Q\rfloor$

$=\lfloor 28 / 14\rfloor$

$=2$

Table 4: Jobs Table with burst times for Proposed Approach

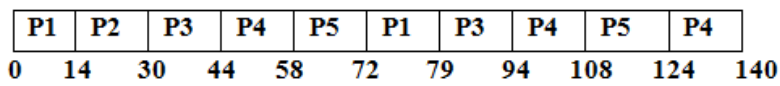

Fig 5: Gantt chart using Proposed Approach

Average Turnaround Time

$=(79+30+94+140+124) / 5$

$=467 / 5$

$=93.4$

Average Wait Time

$=((79-21)+(30-16)+(94-29)+(140-44)+(124-30)) / 5$

$=(58+14+65+96+94) / 5$

$=65.4$

Number of Context switches $=9$.

\subsection{Example 3}

Table 5: Jobs Table with burst times for Conventional Round Robin Algorithm

\begin{tabular}{|l|l|l|l|l|l|l|l|l|l|}
\hline P1 & P2 & P3 & P4 & P5 & P1 & P2 & P3 & P4 & P5 \\
\hline 0 & 4 & 8 & 12 & 16 & 20 & 24 & 28 & 32 & 36
\end{tabular} 40

\begin{tabular}{|l|ll|l|l|l|l|l|l|l|l|}
\hline P1 & P2 & P3 & P4 & P5 & P1 & P2 & P3 & P4 & P5 \\
\hline 40 & 44 & 48 & 52 & 56 & 60 & 64 & 66 & 70 & 74 & 76
\end{tabular}

\begin{tabular}{|l|l|l|l|l|l|}
\hline P1 & P3 & P4 & P3 & P4 \\
\hline 76 & 79 & 83 & 87 & 88 & 94 \\
\hline
\end{tabular}

Fig 6: Gantt chart using Conventional Round Robin Algorithm

4.3.1 Calculations as per Classical Round Robin Algorithm:

Average Turnaround Time $=(79+66+88+94+76) / 5$ 
$=403 / 5$

$=80.6$

Average Wait Time

$=((79-19)+(66-14)+(88-21)+(94-26)+(76-14)) / 5$

$=(60+52+67+68+62) / 5$

$=309 / 5$

$=61.8$

Number of Context switches $=24$.

\subsubsection{Calculations as per proposed approach}

Calculation of TQ:

$\operatorname{Max}(\mathrm{BT})=26$

$\operatorname{Min}(\mathrm{BT})=14$

$\mathrm{C}=\lceil([\operatorname{Max}(\mathrm{BT})-\operatorname{Min}(\mathrm{BT})] / 2)\rceil$

$=\lceil(26-14) / 2\rceil$

$=\lceil 12 / 2\rceil$

$=6$

$\left.\mathrm{Z}=\left\lfloor\sum(\mathrm{BT}[\mathrm{Pi}]) / \mathrm{N}\right)\right\rfloor=18$

As $\mathrm{C}<\mathrm{Z}$,

Therefore $\mathrm{TQ}=6$

Calculation of $\mathrm{K}$

$$
\begin{aligned}
& \mathrm{K}=\lfloor Z / T Q\rfloor \\
& =\lfloor 18 / 6\rfloor \\
& =3
\end{aligned}
$$

Table 6: Jobs Table with burst times for Proposed Approach

\begin{tabular}{|c|c|c|c|}
\hline $\begin{array}{c}\text { Comparison } \\
\text { Parameter } \\
\text { Name }\end{array}$ & $\begin{array}{c}\text { Conventional } \\
\text { Round Robin }\end{array}$ & $\begin{array}{c}\text { Proposed } \\
\text { Approach }\end{array}$ & $\begin{array}{c}\text { Improvement } \\
\text { observed }\end{array}$ \\
\hline $\begin{array}{c}\text { Average } \\
\text { Turnaround } \\
\text { Time (TAT) }\end{array}$ & 66.4 & 59.6 & $\begin{array}{c}6.8 \text { units of } \\
\text { time saved }\end{array}$ \\
\hline $\begin{array}{c}\text { Average } \\
\text { Waiting Time } \\
\text { (WT) }\end{array}$ & 50 & 43.2 & $\begin{array}{c}6.8 \text { units of } \\
\text { time saved }\end{array}$ \\
\hline $\begin{array}{c}\text { Number of } \\
\text { Context } \\
\text { Switches(CS) }\end{array}$ & 17 & 10 & $\begin{array}{c}7 \text { number of } \\
\text { context switch } \\
\text { reduced }\end{array}$ \\
\hline
\end{tabular}

\begin{tabular}{|l|l|l|l|l|l|l|l|l|l|}
\hline P1 & P2 & P3 & P4 & P5 & P1 & P2 & P3 & P4 & P5 \\
\hline 0 & 6 & 12 & 18 & 24 & 30 & 36 & 44 & 50 & 56
\end{tabular} 64

\begin{tabular}{|l|l|l|}
\hline P1 & P3 & P4 \\
\hline 64 & 71 & 80
\end{tabular}

Fig 7: Gantt chart using Proposed Approach

Average Turnaround Time

$=(71+44+80+94+64) / 5$

$=353 / 5$

$=70.6$

Average Wait Time

$=((71-19)+(44-14)+(80-21)+(94-26)+(64-14)) / 5$
$=(52+30+59+68+50) / 5$

$=259 / 5$

$=51.8$

Number of Context switches $=12$.

\section{RESULTS}

In our proposal we are improving the conventional round robin algorithm in two ways, first by dynamically calculating the time quantum by sensing the burst times of the processes and secondly by further increasing the time quantum for the processes which need a fractional greater time than the allocated time quantum cycle(s) to complete their execution. The graphs and tables presented below provide a comparison between the proposed approach and the conventional round robin approach.

Table 7: Comparison table as per Example 1

\begin{tabular}{|c|c|c|c|}
\hline \multicolumn{5}{|c|}{ Time Quantum (TQ) =6 } \\
\hline $\begin{array}{c}\text { Job } \\
\text { Name } \\
\mathbf{P}_{\mathbf{i}}\end{array}$ & $\begin{array}{c}\text { Burst } \\
\text { Time } \\
\text { BT[Pi] }\end{array}$ & $\begin{array}{c}\text { Remaining Time } \\
\text { RT[Pi] } \\
\text { BT[Pi]\%TQ }\end{array}$ & $\begin{array}{c}\text { Number of } \\
\text { Cycles } \\
\text { NQC }\left[\mathbf{P}_{\mathbf{i}}\right]= \\
\left\lceil\mathrm{BT}\left[\mathrm{P}_{\mathrm{i}}\right] / \mathrm{TQ}\right]\end{array}$ \\
\hline P1 & 19 & 1 & 4 \\
\hline P2 & 14 & 2 & 3 \\
\hline P3 & 21 & 3 & 4 \\
\hline P4 & 26 & 2 & 5 \\
\hline P5 & 14 & 2 & 3 \\
\hline
\end{tabular}

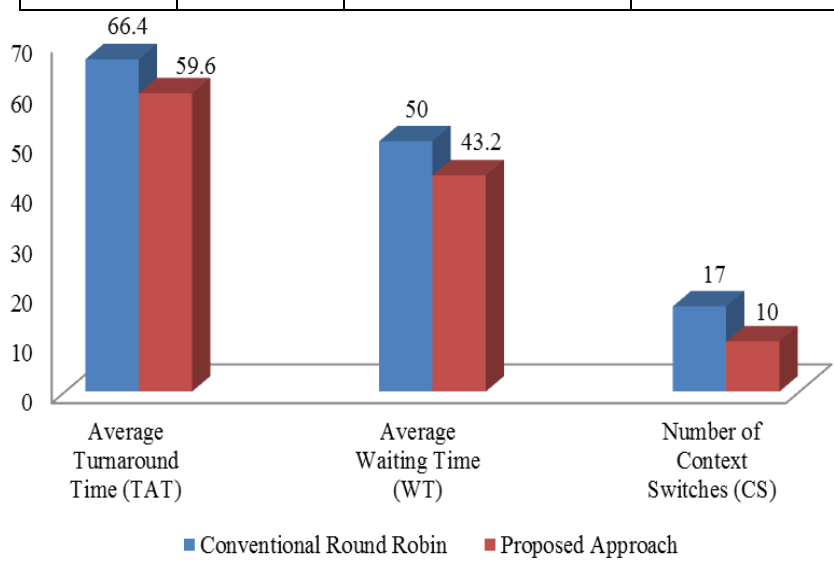

Fig 8: Comparison graph for Example 1
Table 8: Comparison table as per Example 2 


\begin{tabular}{|c|c|c|c|}
\hline $\begin{array}{c}\text { Comparison } \\
\text { Parameter } \\
\text { Name }\end{array}$ & $\begin{array}{c}\text { Conventional } \\
\text { Round Robin }\end{array}$ & $\begin{array}{c}\text { Proposed } \\
\text { Approach }\end{array}$ & $\begin{array}{c}\text { Improvement } \\
\text { observed }\end{array}$ \\
\hline $\begin{array}{c}\text { Average } \\
\text { Turnaround } \\
\text { Time (TAT) }\end{array}$ & 105.6 & 93.4 & $\begin{array}{c}12.2 \text { units of } \\
\text { time saved }\end{array}$ \\
\hline $\begin{array}{c}\text { Average } \\
\text { Waiting Time } \\
\text { (WT) }\end{array}$ & 77.6 & 65.4 & $\begin{array}{c}12.2 \text { units of } \\
\text { time saved }\end{array}$ \\
\hline $\begin{array}{c}\text { Number of } \\
\text { Context } \\
\text { Switches(CS) }\end{array}$ & 13 & 9 & $\begin{array}{c}4 \text { number of } \\
\text { context switch } \\
\text { reduced }\end{array}$ \\
\hline
\end{tabular}

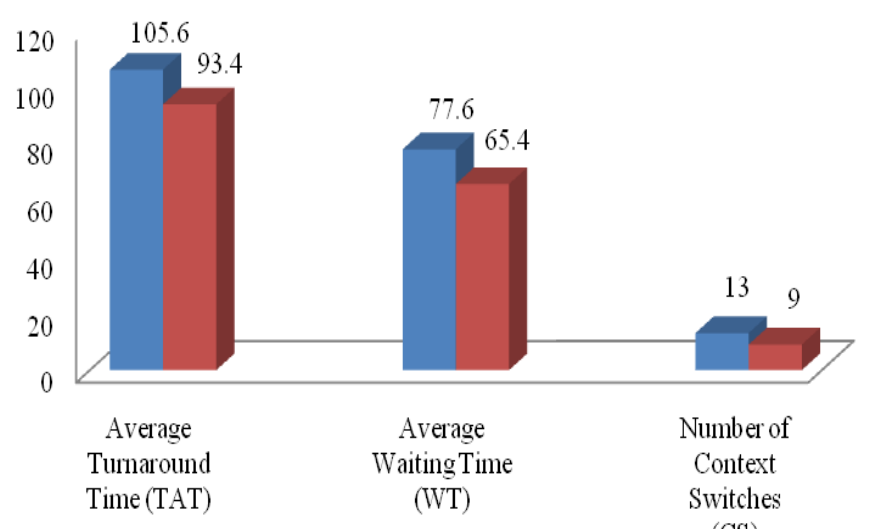

(CS)

- Conventional Round Robin $\quad$ - Proposed Approach

Fig 9: Comparison graph for Example 2

Table 9: Comparison table as per Example 3

\begin{tabular}{|c|c|c|c|}
\hline $\begin{array}{c}\text { Comparison } \\
\text { Parameter } \\
\text { Name }\end{array}$ & $\begin{array}{c}\text { Conventional } \\
\text { Round Robin }\end{array}$ & $\begin{array}{c}\text { Proposed } \\
\text { Approach }\end{array}$ & $\begin{array}{c}\text { Improvement } \\
\text { observed }\end{array}$ \\
\hline $\begin{array}{c}\text { Average } \\
\text { Turnaround } \\
\text { Time (TAT) }\end{array}$ & 80.6 & 70.6 & $\begin{array}{c}10 \text { units of } \\
\text { time saved }\end{array}$ \\
\hline $\begin{array}{c}\text { Average } \\
\text { Waiting Time } \\
\text { (WT) }\end{array}$ & 61.8 & 51.8 & $\begin{array}{c}10 \text { units of } \\
\text { time saved }\end{array}$ \\
\hline $\begin{array}{c}\text { Number of } \\
\text { Context } \\
\text { Switches(CS) }\end{array}$ & 24 & 12 & $\begin{array}{c}12 \text { number of } \\
\text { context switch } \\
\text { saved }\end{array}$ \\
\hline
\end{tabular}

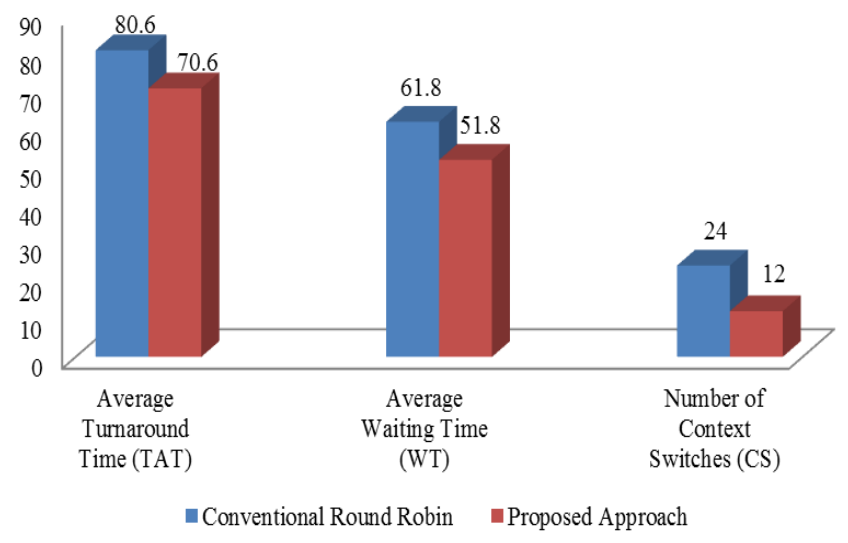

Fig 10: Comparison graph for Example 3

\section{CONCLUSIONS}

From the above results it can be concluded that the proposed approach shows a clear edge over the conventional round robin algorithm. The approach can be further refined using the concept of fuzzy logic in order to calculate the dynamic time quantum.

\section{REFERENCES}

[1] Nayana Kundargi,Sheetal Bandekar,"CPU Scheduling Algorithm Using Time Quantum For Batch System",International Journal Of Latest Trends In Engeneering And Technology(IJLTET), special IssueIDEAS 2013

[2] Himanshi Arora,Deepanshu Arora,Bagish Goel,Parita Jain,"An Improved Scheduling Algorithm",International Journal of applied Information Systems(IJAIS), Foundation of Computer Science FCS, New York, USA, Volume 6- No. 6, December 2013, pp 7-9.

[3] Sandeep Negi,"An Improved Round Robin Approach Using Dyanamic Time Quantum For Improving Average Waiting Time",International Of Computer Applications, Volume 69- No.14, May 2013, pp 12-16.

[4] Rakesh Patel,Mrs.Mili. Patel," SJRR CPU Scheduling Algorithm "International journal of Engineering And Computer Science. Volume 2 Issue 12, Dec.2013 Pp: 3396-3399

[5] Adeeba Jamal,Aiman Jubair" A Varied Round Robin Approach Using Harmonic Mean Of The Remaining Burst Time Of Processes", Special Issue of International Journal of Computer Applications (0975 - 8887), 3rd International IT Summit Confluence 2012 - Pp 11- 17.

[6] Mohd Abdul Ahad,"Modyfying Round Robin Algorithm For Process Scheduling Using Dynamic Quantum Precision", Special Issue Of International Journal of Computer Applications On Issues AND Challenges In Networking ,Intelligence And Computing Technologies, ICNICT 2012, November 2012, Pp 5-10.

[7] C.Yaashuwanth, Dr.R.Ramesh, A New Scheduling Algorithms for Real Time Tasks, (IJCSIS) International Journal of Computer Science and Information Security, Vol. 6, No.2, 2009, Pp: 61-66 
[8] Bashir Alam, Fuzzy Round Robin CPU Scheduling Algorithm, Journal of Computer Science, 9(8) : 10791085, 2013@ 2013 Science Publications.

[9] Lalit Kishor, Dinesh Goyal, Time Quantum Based Improved Scheduling Algorithm, Vol 3 , Issue International Journal of Advanced Research in Computer Science and Software Engineering. 4, April, 2013

[10] P. Surendra Varma, A Best possible time quantum for Improving Shortest Remaining Burst Round Robin (SRBRR) Algorithm, International Journal of Advanced Research in Computer Science and software Engineering, Volume 2, Issue 11, ISSN: 2277 128X , November 2012

[11] Silberschatz, A. P.B. Galvin and G. Gagne (2012), Operating System Concepts, 8th edition, Wiley India

[12] Operating Systems Sibsankar Haldar 2009, Pearson.

[13] D.M. Dhamdhere operating Systems A Concept Based Approach, Second edition, Tata McGraw-Hill, 2006.

[14] Sanjaya Kumar Panda, Sourav Kumar Bhoi, An Effective Round Robin Algorithm using Min-Max Dispersion Measure, IJCSE, Vol. 4 No. 01, pp: 45-53 January 2012

[15] Prof. Bob Walker, Kathryn McKinley, Bradley Chen, Michael Rosenblum, Tom Anderson, John Ousterhout, Paul Farrell/Steve Chapin Operating system lectures notes http://www.cs.kent.edu/ farrell/osf03/ oldnotes/L06.pdf

[16] Aashna Bisht, Mohd Abdul Ahad, Sielvie Sharma, "Enhanced Round Robin Algorithm for Process Scheduling using varying quantum precision" ,Proceedings of ICRIEST AICEEMCS, $29^{\text {th }}$ December, 2013.pp 11-15.

[17] R. N. D. S. S Kiran, Polinati Vinod Babu, B. B. Murali Krishna,Optimizing CPU Scheduling for Real Time Applications Using Mean-Difference Round Robin (MDRR) Algorithm, ICT and Critical Infrastructure: Proceedings of the 48th Annual Convention of Computer Society of India- Vol I, Advances in Intelligent Systems and Computing Volume 248, 2014, pp 713-721. Springer International Publishing Switzerland, 2014.

[18] M.H. Zahedi, M. Ghazizadeh, M. Naghibzadeh, Fuzzy Round Robin CPU Scheduling (FRRCS) Algorithm, Advances in Computer and Information Sciences and Engineering, 2008, pp 348-353, Publisher Springer Netherlands,

[19] Bin Nie, Jianqiang Du, Guoliang Xu, Hongning Liu, Riyue Yu, Quan Wen, A New Operating System Scheduling Algorithm, Advanced Research on Electronic Commerce, Web Application, and Communication, Communications in Computer and Information Science Volume 143, 2011, pp 92-96, Publisher Springer Berlin Heidelberg 